\title{
Studying Illumination and Cultural Heritage
}

\author{
J. Happa and A. Artusi
}

\begin{abstract}
Computer graphics tools and techniques enable researchers to investigate cultural heritage and archaeological sites. They can facilitate documentation of realworld sites for further investigation, and enable archaeologists and historians to accurately study a past environment through simulations. This chapter explores how light plays a major role in examining computer-based representations of heritage. We discuss how light is both documented and modelled today using computer graphics techniques and tools. We also identify why both physical and historical accuracy in modelling light is becoming increasingly important to study the past, and how emerging technologies such as High Dynamic Range (HDR) imaging and physicallybased rendering is necessary to accurately represent heritage.
\end{abstract}

Key words: Light, Illumination, Rendering, High Dynamic Range Imaging, Cultural Heritage.

\section{Introduction}

The application of empirically grounded digital capture of our material culture and visualization is becoming increasingly important for preservation, educational and research purposes. These digital surrogates are used by academics, industry, museums and the media to represent the physical appearance of our cultural heritage throughout time. As digital technologies advance, there is a growing need for techniques to represent real-world historical sites and materials, as well as to investigate ways to better share this captured and subsequently interpreted data.

Computer graphics enables researchers to document and investigate cultural heritage and archaeological sites using computer-based models. Specifically, we use computer graphics for the creation, storage, maintenance, analysis and dissemina-

Jassim Happa, Information Security Group, Royal Holloway, University of London, UK. · Alessandro Artusi, DeepCamera MRG Group, RISE Ltd, Nicosia, Cyprus. 
tion of computer-based (oftentimes referred to as virtual or synthetic) representations of real-world objects in order to facilitate archaeological study. A digital surrogate is a virtual representation of a real-world object that may be used to gain insight into its function and historical relevance. Through simulations in virtual environments, we can study and propose hypotheses about an object, and ask questions related to how it, or a site was used in the past, and how it may have changed over time. These digital surrogates need to be based on existing evidence and expert interpretation available today $[7,39,25]$.

An important aspect of creating digital surrogates to study the past is their appearance, both today and in the past. Light and illumination both play essential roles in the appearance of objects. Light is made up of photons, an elementary particle, that exhibits properties of both particles and waves. As light hits the retina, it is interpreted by the Human Visual System (HVS) to make up what we ultimately perceive as vision. Visible light makes up a small part of the electromagnetic radiation spectrum: between 380 (violet) to 780 (red) nanometres. Colour is a perceived characteristic of light: as light is reflected off an object, hits the eye and is then processed by the HVS. Illumination is the particular use of light to achieve a practical or aesthetic effect. Studying the use of colour, light and illumination can reveal insight into the past $[10,19,49,25]$.

In digital documentation of heritage, light can be captured to a certain degree of accuracy and precision which relies on empirical data acquisition technologies such as cameras and other light measurement equipment. In simulation however, we need to use a light model to describe behaviour of light to accurately model it. We can achieve so different degrees of accuracy. The more accurate and precise the model, the more correct the output of our simulation. The term 'modelling light' is used here to describe properties and behaviour of light, in particular how light propagates from its source and throughout virtual scenes. This is often referred to as physically-based lighting.

Whether capturing or recreating historical light, we also need to consider historically-based information to be present. This must be based on evidence available today, as well as expert opinion and historical sources. Examples of historicallybased input includes for instance a description of how a light source 'was used in the past' as it can affect the illumination in the scene. Furthermore, all resources used (both technical and historical) and the decision-making process must be well documented, in a reproducible manner, otherwise the scientific merit of any output of that simulation will remain questionable.

The field of computer graphics has tackled the challenge of documenting and modelling light for decades, but it is only in the last 20 years that graphics has accelerated its uses to aid historical research [26]. The purpose of this chapter is to provide readers with an overview of how and why documenting and modelling uses of light is becoming increasingly important when documenting real-world heritage. The chapter describes how light in computer graphics is used to study illumination of heritage through in-situ documentation using High Dynamic Range (HDR) imaging and recreating illumination using physically-based rendering. Readers interested in this subject should also be aware of the existence of Reflectance Transformaton 
Imaging (RTI) [?, 26, 21, 33]: a set of imagine techniques aimed to help viewers interactively display objects under varying, user-defined lighting conditions to reveal surface details. The purpose of this chapter however is to examine tools that help facilitate examination of heritage related to study of illumination.

\section{High-Fidelity Rendering and Display of Heritage}

High-fidelity rendering and display of cultural heritage involves the accurate physical and historical documentation (or modelling) of geometry, materials and light to the highest accuracy and precision possible with present day technologies and methods. This allows experts to confirm or disprove assumptions about the past with greater scientific confidence through simulation, and allows new hypotheses to transpire. It should be noted however, that it is only possible to claim any high-fidelity rendering to be scientific best-guesses as simulations or predictions, because it is not possible to prove that the output of these simulations represents the past entirely correctly.

Events in history contribute to changes in appearances of heritage. Structural alterations in the environment (e.g. objects being added or removed), ageing and weathering can alter the physical shape of an object, but also visibly alter the material's light interaction properties from chemical, mechanical and biological processes [20]. Over time, the way light is used by people in the scene change as well. Understanding the relationship between geometry, materials and light in a scene helps build a foundation to investigating sites throughout history using simulations and documentation better.

\subsection{Representing Geometry, Material and Light}

To understand how light is modelled in virtual environments; it is necessary to understand what light can interact with. A distinction is made between two and three-dimensional spaces. Image space assumes $2 \mathrm{D}$ data - an array of pixels that make up an image, projected onto a computer display. These pixels can be regarded as 'samples' of a real or virtual scene, and these samples are what we intend to study. Real scenes can be documented using film or digital cameras. Virtual scenes however, are generally made up of three constituent components: geometry (shape), materials (surface appearance) and light (a distribution of electromagnetic radiation in the scene emanating from one or more light sources). Different types of geometry data structures can be used to mathematically describe shapes. The most commonly used data structure today is polygons. Each polygon is a planar (flat) surface that is defined by a series of boundary points known as vertices. Once all boundary points of a planar surface have been added (typically as a triangle), a surface can be coloured, see Figure 1. 
Many connected polygons that share vertices can make up more complex objects (often referred to as a 'mesh'). Objects appear more detailed if more polygons are used to represent the geometry. Once an object has been formed from a series of points and polygons defining their surfaces, it is necessary to determine the colour of the surface. Material modelling helps us achieve this. Materials describe two aspects of virtual objects surfaces: their intrinsic colour (informally: the colours of the surface "as they are" without affected by light, also known as the surface 'albedo') and their light reflectance functions [34] (i.e., how incoming light will interact with the surface from any incoming angle). Materials (colours and functions) are assigned to surfaces, often as a 2D image (known as a texture map) mapped onto the 3D surface.

Real-world light sources can be described as a function of shape, position, direction, colour (or wavelength), time, polarisation (as light is considered both a particle and a wave, waves oscillate with more than one orientation) and phase of light (waves also have amplitudes). However, modelling light in virtual scenes typically ignores the latter three components and simplifies the light model to Geometric Optics [11]. In the geometric optics model, light is assumed to be travelling in straight lines (as rays), in a vacuum and one ray cannot interfere with another.

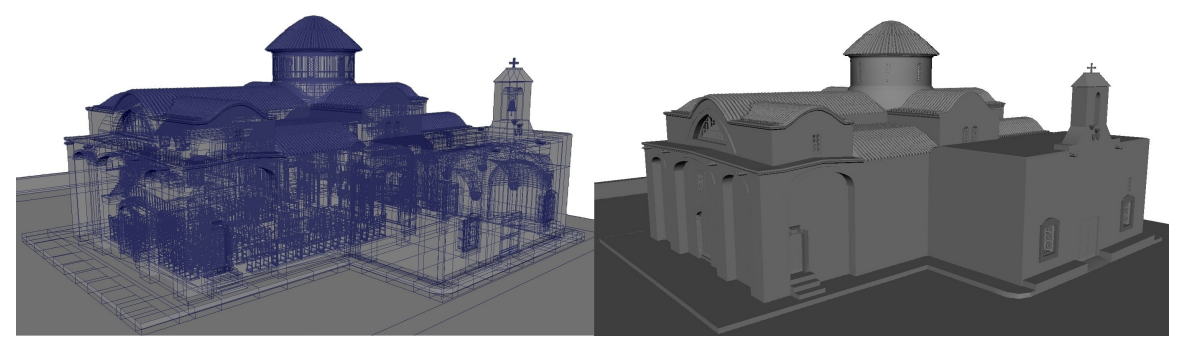

Fig. 1 A flat shaded 3D model example of the exterior and interior of Panagia Angeloktisti, a Byzantine church on Cyprus. Once the points have been defined (left), we can identify which make up the surfaces of objects in the scene (right).

Light-transport simulation is the problem of evaluating how rays intersect with surfaces given a scene configuration, i.e. how geometry, materials and light sources are laid out. Light interacts with materials typically in one of three ways: reflection (e.g. opaque surface such as bricks or stone), transmission (e.g. glass or water) and absorption. The amount of light that is reflected, transmitted and absorbed depends on the reflectance function of the material. While geometric optics generally does not describe visible phenomena that may happen at a quantum or wave level, it is still capable of describing most visual phenomena possible to observe using most camera equipment today. In order to represent colours in images as they appear on screen, we make use of colour models (also known as colour spaces), such as $R G B$ (here colours can be expressed as a combination of three colour primaries that are Red, Green and Blue)[54]. 


\subsection{Capturing Light}

Researchers can use experimental archaeology techniques to recreate objects (that no longer exist) in the real world. In the case of light sources, it is undesirable to use authentic light sources (if they still exist), but it possible to recreate past light sources in the physical world (using the same ingredients and processes), capture the output of that light source digitally, and then use the samples to light a virtual scene. Examples include for instance:

- Roussos [41] presented a reconstruction of a section in the Knossos palace, a Bronze Age archaeological site on Crete. Perceptually realistic and computationally efficient flickering of flames were simulated with his technique, although the approach is limited to small flames from candles.

- Devlin and Chalmers [18] for instance recreated lighting conditions of Roman frescoes in Pompeii by using the same ingredients and processes to create light sources as the Romans in the physical world, then measured the colour profile of the flame using a spectroradiometre (a device used to measure the spectral power distribution of a sample) to study the Roman motivations for light in Roman frescoes and the visual perception of this blue colour under a variety of different light source conditions.

- Sundstedt et al. $[49,42]$ presented colourful Egyptian hieroglyphics viewed under sesame-oil candle, highlighting that in such case (also used during ancient Egypt) the blue paint appears almost green. The interior was modelled to allow exploration and reconstruction of hieroglyphics under contemporary lighting as well for comparison. Properties of real sesame-oil lamps were measured for the reconstruction in a similar fashion to Devlin and Chalmers work.

- Moullou et al. [32] investigated lighting conditions in antiquity houses to assess the photometric basis of studies focusing on ancient lighting. The authors demonstrate an experimental approach to learning about the photometric properties of ancient lamps placed in their appropriate positions. The authors deduced that appropriate positioning of light sources in ancient Greek houses would allow for enough light to read, even in nocturnal conditions.

Another technique that allows acquisition of dynamic range of light available in the real-world is called High Dynamic Range (HDR) Imaging [17]. Once acquired, this light data (now captured at a higher precision) can be used to re-light or study heritage and their illumination based on real-world data capture [27, 14, 23].

\subsection{Modelling Light}

When applying graphics to heritage, parameters of all scene objects (including light sources) carry some semantic meaning in their historical interpretation. The key challenge to using and studying light for historical study is that we fundamentally care about correctness in the simulation and reproduction of light (whereas in many other 
fields if it looks "believable" to most observers then it is satisfactory). For instance, the definition and placements of a light source carry no contextual meaning for general-purpose rendering (i.e., only the fact that the light source is defined properly matters, not necessarily its position). However, in studying history, the placement, its ingredients matter for historical interpretations.

Light is commonly modelled as a geometric object that emits colour with an intensity. The metrics used to express intensity and perceived colours and light are taken from the physical sciences, in particular Radiometry: the field that studies measurement of electromagnetic radiation, including visible light and Photometry: the field that studies measurement of electromagnetic radiation in terms of perceived brightness by the human eye [31]. Before we can represent light sources in virtual environments, we are required to measure them in the real-world to better understand how the correct spectral radiance, or colour and power of light can be added to our virtual models of light. Light sources in virtual scenes can take any geometric shape. Common ways to represent light shapes follow below:

- Ambient Light: An ambient light source evenly illuminates the scene in all directions from all directions. This is typically done by simply multiplying all parts the scene with a constant value. This is not an accurate method to illuminate a virtual scene, but is often used in real-time virtual environments (such as video games) where performance of the lighting computation matters more than the correctness of the output. Its intent is to simulate background illumination that does not appear to be emitted from any particular source.

- Point Light: A point light source is a light source definite at a position in space that illuminates in all directions, often used to represent a light bulb. This approach is also not physically correct, but provides a good approximation to localised lighting.

- Spotlight: A spotlight is a projection of light (often as an inner and outer cone), where the inner cone has more intensity than the outer cone. This is the type of lighting which is commonly used on theatre stages.

- Directional Light: A directional light source illuminates the whole scene from a given direction. It is often used to represent distant light sources such as the sun. It is often modelled as not attenuating with distance.

- Area Light: An areas light source is a surface area that emits light. This is typically defined as a piece of geometry, such as a square. Multiple area light sources can be combined to form more complex light source objects.

- Infinite Area Light: An infinite area light source is a representation of the far distant scene, i.e. the backdrop of any scene whose geometry or materials do not affect the rest of the scene. This is often referred to as an environment map (a photograph surrounding the local scene). The technique used to light a scene from an environment map is often referred to as Image-Based Lighting (IBL) [12]. 


\section{Rendering and Light}

Once the scene has been modelled, we need to simulate how the light interacts with the scene to draw the pixels that form an image. This achieved by the rendering equations [29]. Here, the transport intensity of light, from one surface point to another, is defined as the sum of the emitted light and the total light intensity which is scattered in a direction from all other surface points. In heritage research that focus on correctness of images, we require physically-based rendering methods. Most of these can be categorised as being based on one of two techniques: Rasterisation or Ray Tracing [1, 37].

\subsection{Rasterisation}

Typically, rasterisation is used to achieve believable, real-time (more than 24 frames generated per second) rendering. However, many of rasterisation-related methods do not reliably produce predictive results (simulation satisfactory for scientific inquiry). Rasterisation renders images by projecting polygonal-based geometric primitives (often triangles) to the image (that viewers see) using a series of transformations. Rasterisation is the most popular rendering techniques used today due to its widespread support on modern dedicated graphics hardware and real-time performances. Modern computer graphics APIs such as OpenGL [30] and DirectX [9] provide functionalities for programmers to straightforwardly write simple real-time analytical light models using rasterisation (called shader), in which light on a surface is the sum of all its reflectance properties. While the use of shaders provides useful ways to approximate lighting to give believable results, they do not necessarily incorporate highly accurate physically-based lighting. In these cases, the shading of surfaces is simply calculated as sum of different terms, and then added to make up the final output of the appearance of an object surface. Simplified, this can be written as:

Shading of a point $=$ ambient light + diffuse light + specular light + emission

Each term expresses the contribution of light onto the objects of the scene. The ambient term is (almost always) a constant value that represents some idea of background (ambient) lighting in the scene. The diffuse term describes how light is reflected in all directions equally. While completely diffuse reflections do not happen in reality, it is a straightforward approximation of representing matte objects such as building bricks, sand or carpets. The specular term describes how shiny a surface point should be. In rare cases, in which a material emits light, an "emit" term can be added. 


\subsection{Ray Tracing}

Ray tracing methods [53] tend to focus on delivering physically accurate images, but often at increased computational costs to ensure images are indeed accurate. Ray tracing based approaches are often implemented for Offline rendering (requiring more than one second to compute an image), and are used to synthesise images of highest possible fidelity.

Ray tracing methods for rendering was originally based on a ray casting method (see Appel [2]), which check whether a ray intersects a surface or not. Ray tracing image synthesis is normally done in the reverse order of how our eyes operate. Rays are shot from the eye and an image is formed based on the information accumulated from the various sample rays shot into the scene [43].

Ray tracing is made up of three main components: ray generation, ray intersection and shading. First, a ray is generated for each stage of the path the light has to take. Second, using a point-to-surface intersection test (ray casting), it is possible to determine the closest intersection point visible from a point in a direction. Third, through shading, the specific ray tracing algorithm implemented determines pixel colour based on information from the surface point in the prior intersection test (Figure 2).

The original ray tracing approach simulates light propagation by recursively tracing rays into the virtual scene (i.e. keep sending rays to traverse the scene over and over again). At each intersection, a shadow ray is shot towards the light (shortest distance) to determine whether the intersection point is in shadow or not, see Figure 2. Recursion of all steps in the scene allows for computation of light propagation. These steps are repeated and results are accumulated, and then averaged for as long as required to generate a satisfactory image. Path tracing is a well-known extension to ray tracing that is used for physically-based simulation by evaluating light-surface interactions as well. It was developed by Kajiya [29], and is today often used as a benchmark to assess performance of new techniques.

\section{High Dynamic Range Imaging}

Visual documentation enables scholars to refer to particular sections or entire objects in greater detail than words can express. When a person is viewing a site not in-situ (e.g. in front of a computer monitor), key surface and subsurface information may be clipped if there is a high range of light; anything from 500:1 (standard non-LED LCD display today) to 1000:1 (LED-LCDs) to 200,000:1 (High Dynamic Range displays) and above. This contrast ratio expresses the relative range of the brightest colour (white) to that of the darkest colour (black) that the image or monitor is capable of producing.

Significantly wider ranges of light exist in the real world. Humans are capable of seeing a large range of light intensities ranging from daylight levels of around $10^{8} \mathrm{~cd} / \mathrm{m}^{2}$ to night light conditions of approximately $10^{-6} \mathrm{~cd} / \mathrm{m}^{2}$. On the other hand, 


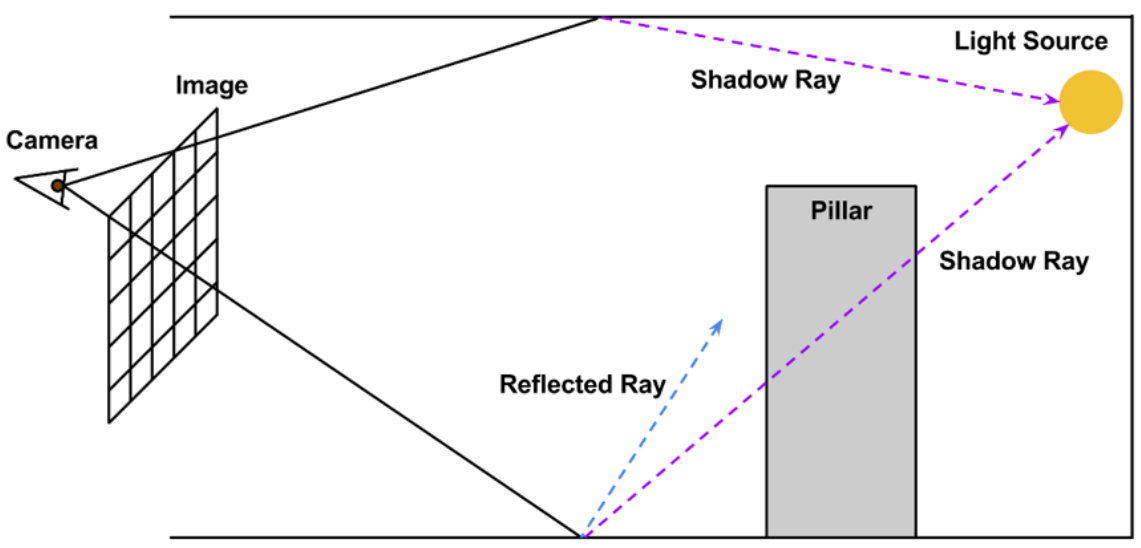

Fig. 2 Classical ray tracing example. Rays are shot into the scene and recursively traversed back to the light source. At each intersection point a shadow raw is cast to check if any objects exist between the ray-surface intersection and a light source.

today we require capturing and displaying larger dynamic range then the one offered previously, i.e., wide colour gamut REC.2020 [55], HDR+ 10-bit support for TV technology, compression technology for HDR content as JPEG XT [4, 40]. However, still the captured and visualized bit-depth is often not enough for conveying the realistic experience of a human watching the same cultural artefacts in the realworld environment. In order to fully achieve this goal we need to capture this large dynamic range, referred to as HDR of luminance ${ }^{1}$, manipulate and visualize it for increasing the reproduction of level of realism in any image output. We can identify as main advantage of HDR imaging in the context of heritage documentation that the scene details, which may have relevance for its interpretation, can be seen in areas that are otherwise obfuscated by darkness, or clipped away in too bright sections. The added information may aid scholars extrapolate how illumination affects a building or object today, and help them discuss how illumination may have been different in the past.

Subtle phenomena such as slow-moving phenomena, such as participating media (e.g. sunbeams) may also be recorded using HDR still photography, as shown in Figure 3. On the other hand, complex dynamic scenes, in which objects are moving fast needs to be recorded using HDR video acquisition solutions.

\subsection{Digital Camera Content Acquisition}

In addition to a traditional photographic record, still images through digital photography can be edited to create texture maps for virtual 3D models. The advent of digital

${ }^{1}$ The intensity of light emitted from a surface per unit area in a given direction. 

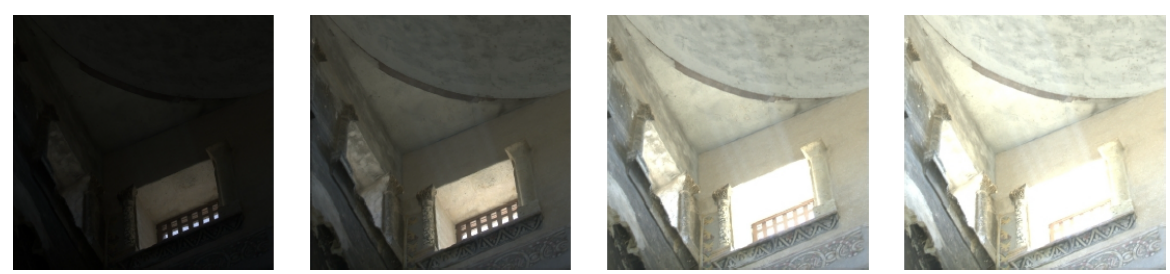

Fig. 3 Participating media (sun beams) at the Red Monastery in Egypt. The figure shows multiple exposures, from low to high exposure. We see that on lower exposures, the camera does not capture the sunbeams. HDR imaging captures all these ranges and assemble them into a single photograph for future examination.

photography has pushed cameras to reach greater bit-depth and image resolutions. Today, high-quality digital cameras are available with sensors capable of capturing 12-16 bits per colour channel. However, lower-quality digital cameras exist that are equipped with less expensive, lower-performing hardware with precision limited to 10 bits or even lower. Moreover, in many application there is the need to acquire much large dynamic range then the one covered by 12-16 bits sensors.

In all these situations, only a small subset of the available dynamic range of the scene can be captured, resulting in overexposed and underexposed areas of the acquired image. To overcome this limitation, one can capture different portions of the dynamic range of the scene by varying the exposure time (multi-exposure) $[3,6]$. Starting from these images, we can reconstruct the HDR content of the originals scene, by first aligning each image to each other (registering), then estimating their camera response function, e.g., mapping input luminance values received by the camera sensor to pixel values. This is useful, because it will allows to estimate the physical quantities of the scene from the acquired images. The final step is to weight the contribution of each image at a given position to the final HDR sample value $[3,6]$. A common concern with the multi-exposure method is the misalignment of the images, either due to movements in the scene or by the camera itself [35]. Merging such images without further processing results in ghosting artefacts in the HDR output.

Video cameras allow for documentation of virtual archaeology from consecutive frames of images, and a minimum of 24 Frames-Per-Second (FPS) is necessary for the human eye to seamlessly interpret the images as natural motion. Video and singleimage content both have advantages and disadvantages. Still photography allows for more control over the camera and its settings. Furthermore, planning needs to be an important part of the data acquisition pipeline. Most concepts from single image photography translate directly to video. The primary difference is the addition of temporal data. Motion blur due to fast motion can be interpreted correctly by the human eye in video. Temporal data makes video recording helpful in delivering a sense of spatial awareness of the environment, more than single photograph often can provide, especially if the camera moves in physical space [23].

The addition of HDR video content is useful in historical study for several reasons. Firstly, the extended light range collected is valuable in the pursuit of understanding 
and recreating the past through experimental archaeology. Secondly, it allows for temporal data acquisition of light. This enables documentation and investigation of movable light sources in areas in deep shadow or intense sunlight, colour properties of artificial light sources as well as the interplay of light through participating media. This accurate data can be reused for virtual archaeology applications. Thirdly, video is a significantly faster approach to record data compared to traditional photography. Simplifying the data acquisition pipeline enables more measurements or video segments to be captured, in addition to reducing the potential of human error in data collection [23].

\subsection{HDR Content Visualization}

The native visualization of HDR content is limited by the capabilities of the display device. Despite the fact that the current technology on the market can guarantee high contrast ratio, this is achieved by lowering the black level. However, the peak luminance remains limited, restricting the available dynamic range for bright images [6]. Even with enhanced contrast, many display panels offer only a limited precision of 8 or, at most, 10-bits per colour channel, and not all of them support a wide colour gamut.

Tone mapping is a process that compresses the dynamic range of an input signal to that available by the display while keeping the visualization convincing. Tone mappers can be roughly classified into global and local approaches. The former applies the same tone curve on the all image pixels. The latter takes the spatial position and its surrounding into account; with that, local operators can take advantage of known effects of the HVS such as local eye adaptation to the luminance. While the former is simple and efficient, it may fail to reproduce details in high contrast image regions. Although the latter can reproduce details in such regions better, it often comes at the cost of increased complexity and computational time; it may also introduce artefacts around edges [6].

Typically tone mapping is focusing on one dimension, e.g., luminance, while colour is mainly mapped keeping the original colour ratio of the input HDR image. This generates two major drawbacks. First, appearance effects are often ignored, leading to images which may appear poorly or too saturated. Second, such a tone mapper may not guarantee that all the sample values of the tone mapped image are within the available colour gamut of the target display. Even though the output luminance may be reproducible by the display, the chrominance may fall out of the available gamut, resulting in clipping of extreme colours. This clipping may again introduce hue shifts. To improve the saturation of the tone mapped image, a simple solution is to introduce an adjustable parameter that allows the overall saturation of the tone mapped image to be controlled [3].

To reduce hue and lightness shifts, one may work with perceptual uniform colour space to separate the colour appearance parameters such as saturation from hue and lightness. This will allow modifying the saturation of the tone mapped image 
to match the saturation of the input HDR image while hue and lightness of the tone mapped image will remain untouched [5]. Other approaches exploit the use of colour appearance models and extend the concept of gamut mapping of the HDR content [44]. The former approach guarantees the matching of the colour appearance attributes between the input HDR and the tone mapped images. The latter ensures that all the tone mapped pixels are within the colour gamut of the display, minimising the hue and luminance distortion.

\subsection{Image-Based Lighting (IBL)}

The emergence of HDR has made it possible to extend the use of capturing HDR photographs to capture radiance maps, i.e. use HDR photographs as light sources for a scene. Such images are referred to as Light Probes or Environment Maps and their application is often referred to as Image-Based Lighting (IBL) [12]. Instead of using synthetic light sources, it is possible to use sampled light values from HDR images mapped onto a sphere that acts as a sky dome to represent the distant illumination. This is useful in order to relight synthetic objects that occupy the same location as the light probe. There are several methods of capturing these HDR environment maps. Debevec [12] details the use of a mirrored sphere. Stumpfel et al. [48] discuss the capturing process of HDR hemispherical image of the sun and sky by using an SLR camera with a $180^{\circ}$ fish-eye lens. In the commercial field, a few companies provide HDR cameras based on automatic multiple exposure capturing. The two main cameras are Spheron HDR VR camera [46] and Panoscan MK-3 [36]. For example, the Spheron HDR VR can capture $26 \mathrm{f}$-stops of dynamic range at 50 Megapixels resolution in 30 min (depending on illumination conditions). Light probe images can be represented in several formats, i.e., mirrored sphere format [12], the vertical cross cube format [15] and the latitude-longitude panoramic representation $[8,15]$. The conversion between these different representations are easily done by using existing software such as HDRShop [28].

The primary concern regarding lighting using light probe images is that a single light probe image is only valid for one point in space. Incident Light Fields allows for capture and rendering of synthetic objects with spatially varying illumination presented by Unger [50]. In virtual archaeology, there are several applications of IBL. It is possible to relight objects with illumination that exists within the site. If the aim is to estimate a light probe image that illuminates objects as they appeared in the past, it is necessary to recreate the distant scene to appear as it once did. This includes switching off modern light sources, remove recent additions to the scene and allow light to be projected in the scene as it would have in the past. Hawkins et al. [27] extend the application of IBL by presenting a photometry-based approach to digitally document heritage artefacts. Each artefact is represented in terms of how it transforms light into images, this is also known as its Reflectance Field. The technique is data intensive and requires a light stage [16] and thousands of photographs of the artefact. 


\section{Case Study: Panagia Angeloktisti}

Panagia Angeloktisti ("Our Lady Built by the Angels" in Greek) is a Byzantine church located in Cyprus. Its origin can be traced back to the sixth century, erected on the ruins of a three-aisled, wooden roofed early Christian basilica [22], similar to the other churches in Cyprus [47]. Its modern day appearance however, pertains largely from its twelfth century iteration. It has undergone many changes throughout history, most noticeably today are:

- A vaulted chapel from the twelfth century added on the north side of the church (now also containing wall-paintings from the fifteenth and sixteenth century).

- A Latin (Gothic) chapel added in 1302 on the south side of the church.

- Throughout the church's history a variety of wall paintings have been added and removed within its space.

- The removal of wall segments in the northern section, including a small tower.

- A modern Gothic-inspired bell tower that was added in the early 1900s only to be removed in 1955 as it was deemed inappropriate based on the history of the rest of the building, by the Antiquities Department of Cyprus. The interior has undergone a variety of changes related to additions of paintings and the placement of interior objects [22].

The church is still in use today. Tourists and visitors frequently enter the building to admire or pray inside the church. Few archaeological drawings of the church (using modern, high precision archaeological tools) exist today. Simple archaeological sketches were made available by Sotiriou [45] and Foulias [22], increasing the need for documentation and preservation.

To measure and model the physical wall geometry, a laser distance meter was used to acquire the dimensions for the $3 \mathrm{D}$ reconstruction stage of the project. This approach was chosen to minimise disrupting the everyday events at the church. Measurement tape was used for more accessible objects such as icons, chairs and ornaments found inside the church. Rulers and measurement tape were added to the photographs to allow distances to be documented. Altogether, data capture sessions lasted for seven days over the span of two-site visits [24].

All measurements were carefully mapped to digitised sketches and drawings of the church. Photographs were taken for texture mapping purposes, as well as for visual references during modelling the geometry of the scene, particularly with such few architectural drawings available. In total, a collection of approximately 10,000 images of the church were taken. They also aided in understanding how light changes in the interior of the church over the course of a day. The church's opening times were from $9 \mathrm{am}$ to $4 \mathrm{pm}$ however, which prevented interior data capture during sunrise and sunset.

All electrical light sources were switched off during data collection and candles were extinguished. Photographs were taken carefully avoiding shadows, specular highlights, caustics and colour bleeding using flash and non-flash photography, and a colour calibration chart to ensure appropriate texture maps could be taken. Large 
pieces of white cloth were used, where appropriate, to diffuse light surrounding icons that were in both shadow and light.

Two types of camera equipment were used for acquiring textures and HDR photographs; conventional digital cameras and a SpheroCam HDR [46] to capture outdoor environment maps. Due to inaccessibility to the roof of the church and no tall buildings in the nearby area all environment maps were acquired at approximately ten metres distance from the main southern entrance. Here, there was a significant enough distance to other large objects, yet it still physically close to the church, enabling us to capture the majority of the sky for relighting purposes during image acquisition. This approach is similar to Debevec's [14] IBL data capture of the Parthenon.

The church was manually reconstructed using a 3D object-modelling tool (Maya) after its modern-day appearance. The interior and exterior church were completed separately, and merged after completion of both versions. This was done as a means to ensure the model was proportional to scale to the rest of the scene. By combining two separate models into one allowed for erroneous data to be corrected in the merging stage. The main interior walls of the church (including arcs, pillars, floor, ceiling and semi-dome) were modelled first based on measurements and sketches. All metal materials were approximated using a Ward BRDF [52], while all matte materials were estimated to be diffuse. All subsequent objects were then positioned and modelled in relation to the main walls, measurements and photographs. Figures 4-6 show the final output of Panagia Angeloktisti, most of which used IBL. In total 24 outdoor environment maps were taken over the course of 14 hours from sunrise to sunset. Path tracing [29] was used to render the scene [24].

As sky model we have used the Preetham's model [38] with the following parameters: Month: 6, Day: 18, Hour: 12:00, Latitude: 34.48'N, Longitude: 33.36'E, Standard Meridian: 0.261, Turbidity (for Preetham's model): 1.0. IBL examples of Panagia Angeloktisti used light captured midday on 18th June 2008.

\subsection{Discussion}

Delivering images that are predictive as opposed to only believable is still challenging however. Even comparing the images side-by-side in Figure 6, it is possible to distinguish between each image from a photograph. A number of several practical challenges need to be overcome, these include:

- Ensuring straightforward and correct measurement of real-world sites.

- Appropriate uses of rendering algorithms.

- Improving correctness of realistic heritage visualization more generally.

Ensuring straightforward and correct measurement of real-world sites is perhaps the greatest challenge, particularly as correct measurement requires physical access, i.e. not only observational access (such as a photograph) but ability and permission to measure objects in more detail (which may lead to physically touching the objects). 


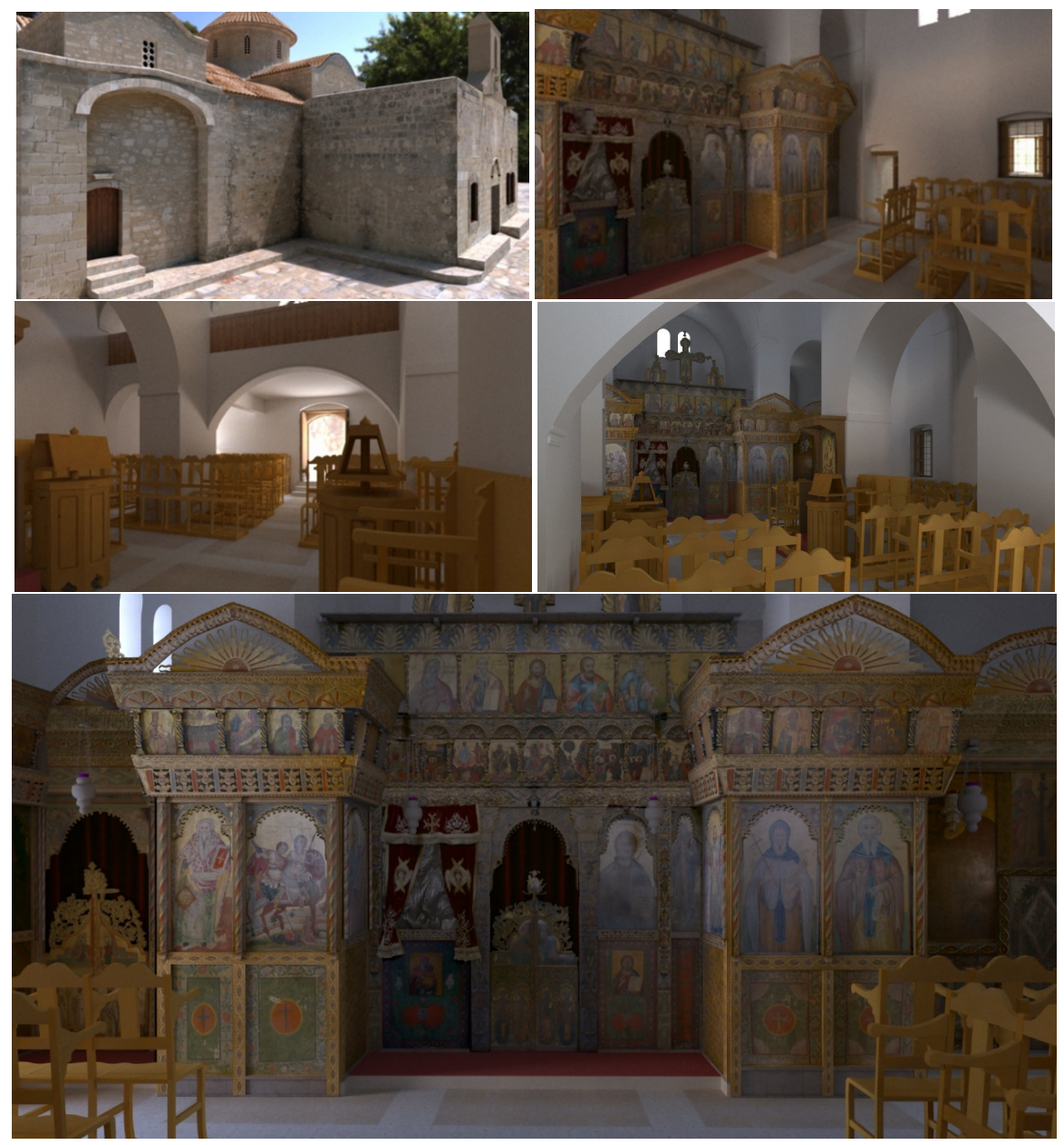

Fig. 4 Path tracing inside and outside Panagia Angeloktisti.

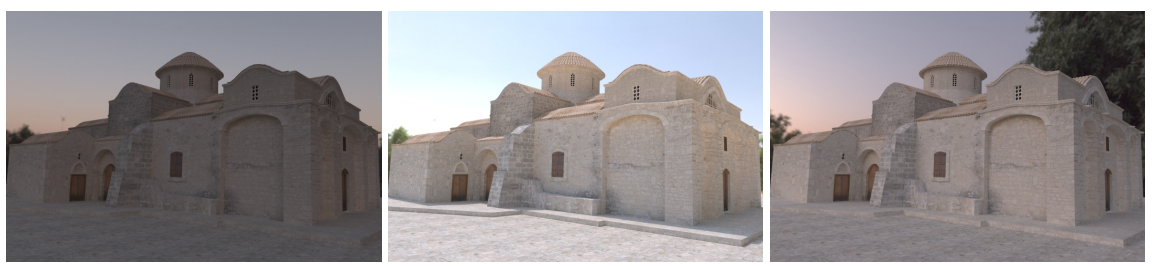

Fig. 5 Daylight simulation. Left: 06:30. Middle: noon. Right: 19:30 


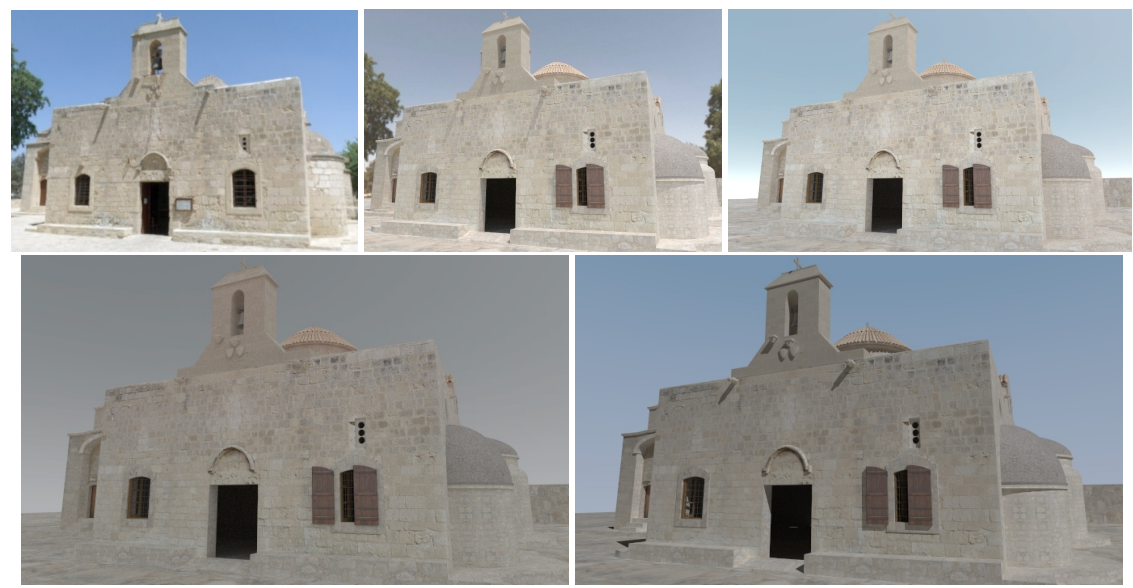

Fig. 6 Top left: photograph. Top middle: Image-based Lighting (captured from site). Top right: Preetham Sky Model [38]. Bottom left: CIE clear sky model. Bottom right: Directional light source mimicking the sun.

As new techniques and technologies develop, it is more likely that computational photography techniques will play a greater role in the future of virtual archaeology, particularly as these methods do not require objects to be touched, only that they are well-lit.

The uses of any rendering algorithm is an essential part in developing appropriate representations of scenes. If interactivity is the primary focus and not necessarily highest accuracy in light computation, real-time rendering approaches can be used to generate believable renditions of the past. However, should light computation be of most value, more physically-based approaches should be used to accurately compute the propagation of light in the scene. While outdoor images were straightforward to compute taking seconds, each interior image of the church took several hours each to compute on a 24-node quad core cluster of computers (from 2008), which makes for impractical times to generate images, even if they are physically-based.

The computer graphics community understands the strengths and weaknesses of existing rendering techniques, but only recently has the use of computer graphics become more frequently used to research past lighting through rendering, a detailed review was recently published by Happa et al. [23]. Historians and archaeologists on the other hand have the knowledge to better understand the contexts and uses of these sites, and can make better judgement about image outputs. To date, efficient interdisciplinary collaboration between technical and non-technical experts has also been described as difficult to achieve[32], and is said to be a contributing factor to why research on modelling light in virtual archaeology has not come further than it already has.

Many virtual archaeology researchers depend on existing implementations often found in commercial solutions. More often than not, third-party solutions are used to model and simulate light. While many of these solutions provide useful graphical 
user interfaces to simplify the development process, almost all of these were made with general-purpose high-fidelity rendering in mind, and not physical or historical accuracy. More importantly, most of these interfaces were not intended for scientific or historical research, but to deliver believable renditions that have some basis in physically-based methods. Commercial rendering solutions are problematic from a research perspective. This is because it is difficult to reproduce results that these solutions output. For instance, if the developer changes their software, it might not be possible to recreate the same image in the future. It is also near impossible for researchers reproduce simulation results without complete access to the mesh and all scene configurations that the other researcher made use of.

Any simulations that are produced using such rendering systems are not reproducible, and therefore not appropriate from a scientific researcher's standpoint. Users of these systems are not made explicitly aware of these underlying issues, and this is a significant problem to the research community as a whole. In the case of Panagia Angeloktisti, three different renderers were used to synthetise images during the project, including Maya's own renderer Mental Ray, Radiance [51] and a heavily modified version of PBRT [37].

\section{Conclusion}

This chapter has discussed the uses of documenting and modelling light in for historical research, including the uses of physically-based light and historically-based light. We examined how incorporating historical knowledge into virtual scenes is crucial for historical research. Historically-based input must be based on evidence available today, including expert opinion. Another crucial aspect of any investigation using computer graphics is that all decisions and processes involved must be welldocumented, otherwise the value of any simulation output will suffer greatly. A case study with the use of physically-based and historically-based simulation was also presented.

Computer graphics and the topic of high-fidelity rendering in particular has tackled the problem of simulating light for a number of decades, but only in the recent decade have we identified how important modelling light can aid archaeological research. This chapter serves as an overview to how history research uses physicallybased light simulations to study the past. Using physically-based and historicallybased simulations still has a long way to go before becoming common practice in a scientifically rigorous and reproducible manner.

\section{Acknowledgements}

Our thanks go to the Byzantine Art Gallery for providing permission for us to visit Panagia Angeloktisti, to the caretakers of the church and Andreas Foulias for his help 
on information regarding the church. Our thanks also go to the American Research Center in Egypt (ARCE) and Dr. Elizabeth Bolman for permission to visit the Red Monastery and organising the stay at the monastery. The visit produced 3.

Dr. Artusi work has been partially funded through the the European Union's Horizon 2020 research and innovation programme under grant agreement No 739578 and the Government of the Republic of Cyprus through the Directorate General for European Programmes, Coordination and Development.

\section{References}

1. Tomas Akenine-Möller, Eric Haines, Naty Hoffman, Angelo Pesce, and Sebastien Hillaire. Real-time rendering. AK Peters/CRC Press, 2018.

2. Arthur Appel. Some techniques for shading machine renderings of solids. In Proceedings of the Spring Joint Computer Conference, pages 37-45, 1968.

3. Alessandro Artusi, Francesco Banterle, Tunç Ozan Aydın, Daniele Panozzo, and Olga SorkineHornung. Image content retargeting: maintaining color, tone, and spatial consistency. AK Peters/CRC Press, 2016.

4. Alessandro Artusi, Rafal K Mantiuk, Thomas Richter, Pavel Korshunov, Philippe Hanhart, Touradj Ebrahimi, and Massimiliano Agostinelli. JPEG XT: A compression standard for hdr and wcg images [standards in a nutshell]. IEEE Signal Processing Magazine, 33(2):118-124, 2016.

5. Alessandro Artusi, Tania Pouli, Francesco Banterle, and Ahmet Oğuz Akyüz. Automatic saturation correction for dynamic range management algorithms. Signal Processing: Image Communication, 63:100-112, 2018.

6. Alessandro Artusi, Thomas Richter, Touradj Ebrahimi, and Rafal K Mantiuk. High dynamic range imaging technology [lecture notes]. IEEE Signal Processing Magazine, 34(5):165-172, 2017.

7. Norman I. Badler and Virginia R. Badler. Interaction with a color computer graphics system for archaeological sites. SIGGRAPH, 12:217-221, 1978.

8. James F. Blinn and Martin E. Newell. Texture and reflection in computer generated images. Commun. ACM, 19(10):542-547, 1976.

9. David Blythe. The direct3d 10 system. ACM Trans. Graph., pages 724-734, 2006.

10. Duncan H Brown, Alan Chalmers, and Ann MacNamara. Light and the culture of colour in medieval pottery. In Method and Theory in Historical Archaeology. Pre-printed papers of the Medieval Europe Brugge 1997 Conference, volume 10, pages 145-47, 1997.

11. R. L. Cook and K. E. Torrance. A reflectance model for computer graphics. ACM Trans. Graph., 1(1):7-24, 1982.

12. Paul Debevec. Rendering synthetic objects into real scenes: bridging traditional and imagebased graphics with global illumination and high dynamic range photography. In SIGGRAPH '98: Proceedings of the 25th annual conference on Computer graphics and interactive techniques, 1998.

13. Paul Debevec. Making the parthenon. In 6th International Symposium on Virtual Reality, Archaeology, and Cultural Heritage in Pisa, Italy, 2005.

14. Paul Debevec. High Resolution Light Probe Gallery. http://gl.ict.usc.edu/Data/HighResProbes/, 2006.

15. Paul Debevec, Tim Hawkins, Chris Tchou, Haarm-Pieter Duiker, Westley Sarokin, and Mark Sagar. Acquiring the reflectance field of a human face. In Proceedings of the 27th annual conference on Computer graphics and interactive techniques, pages 145-156. ACM, 2000.

16. Paul Debevec and Jitendra Malik. Recovering high dynamic range radiance maps from photographs. In SIGGRAPH '97: Proceedings of the 24th annual conference on Computer graphics and interactive techniques, pages 369-378, 1997. 
17. Kate Devlin. Perceptual Fidelity for Digital Image Display. PhD thesis, University of Bristol, 2004.

18. Kate Devlin and Alan Chalmers. Realistic visualisation of the pompeii frescoes. Conference on Computer Graphics, Virtual Reality, Visualisation and Interaction in Africa (AFRIGRAPH), pages 43-48, 2001.

19. Julie Dorsey, Holly Rushmeier, and François Sillion. Digital Modeling of Material Appearance. Morgan Kaufmann, 2008.

20. G. Earl, K. Martinez, and T. Malzbender. Archaeological applications of polynomial texture mapping: analysis, conservation and representation. Journal of Archaeological Science, 37(8):2040-2050, 2010.

21. Andreas M. Foulias. The Church of our Lady Angeloktisti at Kiti. Nicosia, 2004.

22. Jassim Happa, Alessandro Artusi, Silvester Czanner, and Alan Chalmers. High dynamic range video for cultural heritage documentation and experimental archaeology. In Proceedings of the 11th International conference on Virtual Reality, Archaeology and Cultural Heritage, pages 17-24. Eurographics Association, 2010.

23. Jassim Happa, Alessandro Artusi, Piotr Dubla, Thomas Bashford-Rogers, Kurt Debattista, Vedad Hulusić, and Alan Chalmers. The Virtual Reconstruction and Daylight Illumination of the Panagia Angeloktisti. In VAST, 2009.

24. Jassim Happa, Thomas Bashford-Rogers, Alexander Wilkie, Alessandro Artusi, Kurt Debattista, and Alan Chalmers. Cultural heritage predictive rendering. In Computer Graphics Forum. Wiley Online Library, 2012.

25. Jassim Happa, Mark Mudge, Kurt Debattista, Alessandro Artusi, Alexandrino Gonçalves, and Alan Chalmers. Illuminating the past: state of the art. Virtual reality, 14(3):155-182, 2010.

26. Tim Hawkins, Jonathan Cohen, and Paul Debevec. A photometric approach to digitizing cultural artifacts. In VAST 2001: Symposium on Virtual reality, archeology, and cultural heritage, pages 333-342, 2001.

27. HDRShop. Example software to research hdri. http://gl.ict.usc.edu/HDRShop/.

28. James Kajiya. The rendering equation. In Conference on Computer graphics and interactive techniques (SIGGRAPH), pages 143-150, 1986.

29. Khronos Group. OpenGL official website. https://www.opengl.org/, 2019.

30. Tom Malzbender, Dan Gelb, and Hans Wolters. Polynomial texture maps. In SIGGRAPH '01, pages 519-528. ACM, 2001.

31. William R. McCluney. Introduction to radiometry and photometry. Artech House, 2014.

32. Dorina Moullou, Evangelos Nikolaos Madias, Lambrou Doulos, Constantinos A. Bouroussis, and Frangiskos V. Topalis. Lighting in antiquity. In M. Kostic (éd.), Balkan Light 2012, Proceedings of the fifth conference, Belgrade, pages 3-6, 2012.

33. M. Mudge, C. Schroer, G. Earl, K Martinez, H. Pagi, C. Toler-Franklin, S. Rusinkiewicz, G. Palma, M. Wachowiak, M. Ashey, N Mathews, T. Noble, and M. Dellepiane. Principles and practices of robust, photography-based digital imaging techniques for museums. In 11th VAST International Symposium on Virtual Reality, Archaeology and Cultural Heritage, September 2010.

34. F.E. Nicodemus, J.C. Richmond, and J.J. Hsai. Geometric Considerations and Nomenclature for Reflectance, volume 160. U.S. Department of Commerce, National Bureau of Standards Report, National Bureau of Standards Washington, DC, 1977.

35. Raisel R. Orozco, Celine Loscos, Ignacio Martin, and Alessandro Artusi. Multiview hdr video sequence generation. In High Dynamic Range Video, pages 121-138. Elsevier, 2016.

36. Panoscan. Panoscan MK-3, Company website. http://www.panoscan.com/, 2002.

37. Matt Pharr, Wenzel Jakob, and Greg Humphreys. Physically based rendering: From theory to implementation. Morgan Kaufmann, 2016.

38. A. J. Preetham, Peter Shirley, and Brian Smits. A practical analytic model for daylight. In SIGGRAPH '99: Proceedings of the 26th annual conference on Computer graphics and interactive techniques, pages 91-100, 1999.

39. Paul Reilly. Towards a virtual archaeology. Computer Applications and Quantitative Methods in Archaeology, pages 133-140, 1991. 
40. Thomas Richter, Alessandro Artusi, and Touradj Ebrahimi. JPEG XT: A new family of jpeg backward-compatible standards. IEEE MultiMedia, 23(3):80-88, July 2016.

41. Ioannis Roussos and Alan Chalmers. High fidelity lighting of knossos. In VAST 2003: Symposium on Virtual Reality, Archaeology and Intelligent Cultural Heritage, 2003.

42. Ivana Rudolfova and Veronica Sundstedt. High Fidelity Rendering of the Interior of an Egyptian Temple. In CESCG, 2004.

43. Peter Shirley and R. Keith Morley. Realistic ray tracing. AK Peters, Ltd., 2008.

44. Elena Sikudová, Tania Pouli, Alessandro Artusi, Ahmet Oguz Akyüz, Francesco Banterle, Zeynep Miray Mazlumoglu, and Erik Reinhard. A gamut-mapping framework for coloraccurate reproduction of hdr images. IEEE computer graphics and applications, 36(4):78-90, 2015.

45. G Sotiriou. The Byzantine monuments of Cyprus, 1935.

46. Spheron. HDR spherical camera, company website. http://www.spheron.com/, 2007.

47. Charles A. Stewart. The First Vaulted Churches in Cyprus. Journal of the Society of Architectural Historians, 2010.

48. Jessi Stumpfel, Chris Tchou, Andrew Jones, Tim Hawkins, Andreas Wenger, and Paul Debevec. Direct hdr capture of the sun and sky. In Proceedings of the 3rd international conference on Computer graphics, virtual reality, visualisation and interaction in Africa, pages 145-149. $\mathrm{ACM}, 2004$.

49. Veronica Sundstedt, Alan Chalmers, and Philippe Martinez. High fidelity reconstruction of the ancient egyptian temple of kalabsha. In AFRIGRAPH '04: Proceedings of the 3rd international conference on Computer graphics, virtual reality, visualisation and interaction in Africa, pages 107-113, 2004.

50. Jonas Unger. Incident Light Fields. PhD thesis, Linköping University, 2009.

51. Gregory J. Ward. The radiance lighting simulation and rendering system. In SIGGRAPH '94: Proceedings of the 21st annual conference on Computer graphics and interactive techniques, pages 459-472, 1994.

52. Gregory J Ward et al. Measuring and modeling anisotropic reflection. Computer Graphics, 26(2):265-272, 1992.

53. Turner Whitted. An improved illumination model for shaded display. In ACM SIGGRAPH Computer Graphics, volume 13, page 14. ACM, 1979.

54. Gunter Wyszecki and Walter Stanley Stiles. Color science, volume 8. Wiley New York, 1982.

55. Ruidong Zhu, Zhenyue Luo, Haiwei Chen, Yajie Dong, and Shin-Tson Wu. Realizing rec. 2020 color gamut with quantum dot displays. Optics express, 23(18):23680-23693, 2015. 